\title{
Motivación y desempeño laboral Proyecto Especial Huallaga Central y Bajo Mayo
}

\author{
Mg. Wilfredo Torres Reátegui \\ wtorres@unsm.edu.pe \\ ORCID: 0000-0001-8811-0703
}

Mg. Cinthya Torres Silva ctorresv@unsm.edu.pe ORCID: 0000-0002-8543-6975

Mtro. José Gabriel Seijas Díaz Jseijasd@unsm.edu.pe ORCID: 0000-0002-7542-2254

\section{Mtro. Roger Ricardo Rengifo Amasifen rrengifoa@unsm.edu.pe ORCID: 0000-0003-0579-6928}

\section{RESUMEN}

Mtro. Karla Patricia Martell Alfaro kpmartella@unsm.edu.pe ORCID: 0000-0002-4416-8740

Para llevar a cabo la investigación se formuló el siguiente objetivo general: Determinar el nivel de relación existente entre la motivación de los colaboradores del P.E.H.C.B.M. y su desempeño laboral - 2014, así mismo se contó con los siguientes objetivos específicos: Conocer cómo se encuentra la motivación de los colaboradores del P.E.H.C.B.M. y Conocer cómo está el estado del desempeño laboral de los mismos. La investigación concluyo que la motivación de los colaboradores de dicha institución es considerada baja, manifestándose en la poca dedicación y esfuerzo de los mismos a la hora de realizar las tareas asignadas, en cuanto al desempeño laboral se identificó que ésta es considerada inadecuada, viéndose reflejado en la presentación de las tareas fuera de tiempo, así como el incremento innecesario de los recursos y la baja productividad, por tal motivo se observa que mientras más problemas internos existan, la motivación de los colaboradores se verá afectada de manera negativa provocando que esta comience a decaer.

Palabras clave: Motivación, Desempeño Laboral, colaboradores. 


\title{
Motivation and job performance Special Project Huallaga Central and
} Bajo Mayo

\begin{abstract}
ABSTRAC
To carry out the research, the following general objective was formulated: To determine the level of relationship between the motivation of the P.E.H.C.B.M. and their work performance - 2014, likewise, the following specific objectives were included: To know how the motivation of the P.E.H.C.B.M. and Know how is the status of their job performance. The investigation concluded that the motivation of the collaborators of said institution is considered low, manifested in the little dedication and effort of them when carrying out the assigned tasks, in terms of work performance it was identified that it is considered inadequate, being reflected in the presentation of tasks out of time, as well as the unnecessary increase of resources and low productivity, for this reason it is observed that the more internal problems exist, the motivation of the collaborators will be negatively affected causing it to begin to decay
\end{abstract}

Keywords: Motivation, Work Performance, collaborator.

Artículo recibido: 15 enero2020 Aceptado para publicación: 18 febrero2020 Correspondencia: wtorres@unsm.edu.pe Conflictos de Interés: Ninguna que declarar 


\section{INTRODUCCIÓN}

Las organizaciones constituyen en el mundo moderno la institución dominante de la sociedad y a través de ellas, las personas pueden alcanzar metas que individualmente no podrían lograr, como producir y distribuir la mayoría de los bienes y servicios que la sociedad necesita para poder subsistir. Además de los conocimientos, habilidades y destrezas que deben poseer los individuos para contribuir con el logro de los objetivos organizacionales, es necesario que el personal esté motivado, es decir, que sientan que su esfuerzo será retribuido mediante recompensas que ellos consideran que satisfacen sus principales necesidades personales y profesionales. Como toda organización, para alcanzar sus objetivos el PEHCBM requiere contar con un equipo de trabajadores altamente motivados, que puedan tener un buen desempeño, para lo cual deben tener altos niveles de satisfacción laboral. Ahora bien, a través de observaciones directas en el PEHCBM, durante el segundo trimestre laboral realizadas por el autor, se pudo detectar algunas situaciones que, son síntomas de que la motivación del personal no se halla en un alto nivel.

Para mejorar la situación que actualmente se presenta en el PEHCBM, es pertinente implementar algunas medidas que permitan superarla, para lo cual es necesario determinar el nivel de satisfacción general de los trabajadores de la institución, e identificar los factores que pueden estar afectando la motivación de su personal. Se pretende conocer cuánta motivación están generando esos factores para proponer iniciativas orientadas a mejorarlos. De esa forma, la investigación da respuesta a las siguientes interrogantes específicas, determinar la motivación que poseen los empleados del PEHCBM, determinar el desempeño laboral de los empleados del PEHCBM.

\section{ESTRATEGIAS METODOLÓGICAS O MATERIALES Y MÉTODOS}

La investigación tuvo un tipo aplicada, de diseño no experimental descriptiva, correlacional, transversal, con una población conformada por 174 colaboradores, se aplicó como técnica la encuesta y el fichaje, siendo el instrumento un cuestionario, los datos cuantitativos fueron procesados y analizados por medios electrónicos, clasificados y sistematizados de acuerdo a las unidades de análisis correspondientes, respecto a sus variables, a través de Microsoft Excel y el programa estadístico SPSS

Los instrumentos pasaron un proceso de validación y confiabilidad en cuanto al contenido de los Ítems del cuestionario aplicado a la población objeto de estudio, los cuales fueron 
desarrollados bajo la fundamentación teórica de las variables investigadas, bajo este contexto, la validez de esta investigación se determinó a través del método estadístico.

\section{RESULTADOS Y DISCUSIÓN}

Tabla 1: Motivación de los colaboradores del Proyecto Especial Huallaga Central y Bajo Mayo

\begin{tabular}{|l|c|c|}
\hline \multicolumn{1}{|c|}{ Escalas de medición } & Frecuencia & Porcentaje \\
\hline Inadecuado & 115 & $66 \%$ \\
\hline Regular & 9 & $5 \%$ \\
\hline Adecuado Total & 50 & $29 \%$ \\
\hline \multicolumn{1}{|c|}{ The } & $\mathbf{1 0 0 \%}$ \\
\hline
\end{tabular}

Fuente: Elaboración propia

Se puede apreciar que el nivel de motivación entre los colaboradores del Proyecto Especial Huallaga Central y Bajo Mayo, es baja, lo cual provoca ciertos conflictos y descoordinaciones a la hora de realizar las actividades programadas dentro de la institución.

Tabla2: Desempeño laboral de los colaboradores del Proyecto Especial Huallaga Central y

Bajo Mayo

\begin{tabular}{|l|c|c|}
\hline \multicolumn{1}{|c|}{ Escalas de medición } & Frecuencia & Porcentaje \\
\hline Inadecuado & 105 & $60 \%$ \\
\hline Regular & 16 & $9 \%$ \\
\hline Adecuado Total & 53 & $30 \%$ \\
\hline \multicolumn{2}{|c|}{$\quad \mathbf{1 7 4}$} & $\mathbf{1 0 0 \%}$ \\
\hline
\end{tabular}

Fuente: Elaboración propia

Se pudo conocer el estado del desempeño de los colaboradores. Este resultado se resuelve debido a que los jefes inmediatos encargados de evaluar a su personal, han identificado que, durante el segundo trimestre del 2014, el nivel de eficacia, eficiencia y efectividad de sus subordinados se ha visto decaída debido a una serie de factores, en cuanto a la eficacia, se obtuvo que los colaboradores para llegar a dar con los resultados esperados hacen uso de demasiados recursos, y que no por ello llegan a cumplir con las mismas. Es por ello que al no realizar los trabajos como se debe, en el tiempo estipulado y haciendo uso adecuado de los recursos, es que no se llega a alcanzar la efectividad dentro de esta institución. 
Tabla 3: Relación entre motivación y el desempeño laboral

\begin{tabular}{|ll|c|c|}
\hline & Correlaciones & Motivación & Desempeño \\
\hline \multirow{3}{*}{ Motivación } & Correlación de Pearson & 1 &, $808^{* *}$ \\
& Sig. (unilateral) & &, 000 \\
& $\mathrm{~N}$ & 174 & 174 \\
& Correlación de Pearson &, $808^{* *}$ & 1 \\
Desempeño & Sig. (unilateral) &, 000 & \\
& $\mathrm{~N}$ & 174 & 174 \\
\hline
\end{tabular}

**. La correlación es significante al nivel 0,01 (unilateral).

Fuente: Elaboración propia

Como se puede apreciar en la tabla $\mathrm{N}^{\circ}$ 3, la correlación de Pearson obtenida en cuanto a la motivación y desempeño de los 174 (siento setenta y cuadro) colaboradores del Proyecto Especial Huallaga Central y Bajo Mayo (P.E.H.C.B.M.), es de 0.808; Este resultado indica que existe un alto nivel de relación entre las variables estudiadas, por tal motivo se observa que mientras más problemas internos existan, la motivación de los colaboradores se verá afectada de manera negativa provocando que esta comience a decaer; Esto a su vez provoca que factores como la productividad y el nivel de desempeño comience a decaer periodo tras periodo. Es por ello que en la presente investigación se acepta la $\mathrm{Hi}$, el cual hace referencia de que existe una alta relación entre la motivación de los colaboradores del P.E.H.C.B.M. y su desempeño laboral durante el segundo trimestre del 2014.

\section{Discusión}

El desempeño dentro de una organización o institución siempre ha sido un factor importante para poder alcanzar los objetivos y metas trazadas, es por ello que se decidió estudiarla para conocer dentro del periodo estipulado, cuan adecuado o inadecuado se encontraba. quien hace mención que el desempeño es un reflejo del esfuerzo que realizan los colaboradores para lograr de manera correcta un determinado trabajo dentro de los tiempos establecidos. Tomando en cuenta lo mencionado y comparándolo con los resultados obtenidos, se puede apreciar que el desempeño dentro de la institución estudiada, es considerado inadecuada puesto que los trabajos no se desarrollan de la manera correcta en los tiempos establecidos y la calidad de su trabajo no es la más efectiva para los estándares que la institución requiere. " Palencia, G. (2008), , en su investigación titulada "Clima Laboral en el desempeño laboral del personal civil de la armada, llegó a la conclusión de que el personal para mejorar su desempeño necesita fortalecer los niveles 
de comunicación interna y recibir incentivos tanto monetarios como reconocimiento personal, pues ésta influye con el clima organizacional donde se desempeñan, con respecto a la investigación se pudo concluir que el personal necesita de mayores incentivos y motivaciones para ejercer un mejor trabajo, así mismo se identificó que el personal necesita administrar y utilizar de manera adecuada los recursos en el menor tiempo. Robbins y Uzcàtegui, Juan (2007), quienes mencionan que sí existe una relación teórica entre ambas variables en estudio, por ende en los resultados obtenidos se encontró que sí existe relación entre ambas, puesto que el personal se muestra desmotivado y por consiguiente su desempeño ha decaído considerablemente en dicha institución esto según lo aportado por los jefes inmediatos de cada uno de los colaboradores, quienes al estar en contacto diario con los mismos se encuentran en la capacidad suficiente como para calificarlos.

\section{CONCLUSIÓN O CONSIDERACIONES FINALES}

\section{Conclusiones}

se acepta como probablemente cierta la hipótesis nula que menciona la existencia de motivación en un nivel bajo, puesto que se ha identificado un nivel bajo de motivación en un $66 \%$ a comparación de un $34 \%$ del personal que manifiesta sentirse motivado, es decir la mayoría de los colaboradores no se sienten impulsados ni direccionados a alcanzar los objetivos planteados por dicha entidad, así mismo se denota una prevalencia de actitud poco entusiasta y participativa para el desarrollo de las tareas encomendadas por sus superiores. Así mismo se identificó que el nivel de desempeño laboral que muestran los colaboradores es considerado inadecuado en un $60 \%$, debiendo evaluar la motivación de los colaboradores de la institución bajo nuevos indicadores que enfoquen de manera directa si su estado actual se debe a factores internos o externos, y en función a ello desarrollar una serie de posibles soluciones.

\section{Recomendaciones}

Evaluar el desempeño laboral de los colaboradores de manera periódica y generar políticas de rotación interna siempre y cuando se conozcan las habilidades de cada uno de ellos para no realizar modificaciones sin fundamento que podrían representar pérdidas económicas, así mismo se sugiere realizar un estudio para determinar otras causas que repercuten sobre el desempeño de los colaboradores, a fin de tener mayor información para generar medidas correctivas a la hora de presentarse los problemas estudiados. Se 
recomienda profundizar los instrumentos de investigación para obtener una información más concreta y detallada de las variables estudiadas.

\section{LISTA DE REFERENCIAS}

Alfaro, R. (2012) "Satisfacción Laboral Y Su Relación Con Algunas Variables Ocupacionales En Tres Municipalidades” de la Pontificia Universidad Católica del Perú.

Alzola, Rubén (2010). “Los Pilares del Desempeño Laboral”. Editorial Mc GRAW HILL. - Segunda Edición, México.

Alzola, Rubén (2010). Las tres dimensiones del desempeño laboral. http://www.alzola.cl/portal/index.php?option=com_content\&view=article\&id=35:74 1-a001-las-tres-dimensiones-del-desempeño-laboral\&catid=6:orange-blue-papers

Coleman y Borman (2000). "Escala de Desempeño cívico y Análisis de la Estructura Empírica del Constructo". Tomado de: http://scielo.isciii.es/scielo.php?pid=S157659622012000300001\&script=sci_artte

Feldman Robert (2012) Psicología para Bachillerato. Editorial Mc GRAW HILL. Primera Edición, México.

Fleitman, Jack. Evaluación Integral para Implementar Modelos de Calidad. C:/Ulsers/USUARIO/Eficacia, Eficiencia y Efectividad-Youtube.mht

Idalberto Chiavenato (2011) Administración de Recursos Humanos - El Capital Humano de las Organizaciones. Editorial McGRAW HILL. - Novena Edición, México.

Machuca Maza, A. (2009) Las Organizaciones del Siglo XXI - Apuntes de Administración General. Editorial San Marcos E.I.R.L. - Primera Edición, Perú.

Marroquín Stefani y Pérez Lorena (2011). “El clima organizacional y su relación con el desempeño laboral en los trabajadores de Burger King”. Universidad de San Carlos. Guatemala. negotiun/ciencias gerenciales-universidad de Zulia, año $3 / n^{\circ} 9$, en Venezuela,

Palencia, G. (2008) “Clima organizacional en el desempeño laboral del personal civil de la armada”. Universidad Nacional Experimental Politécnica de la Fuerza Armada Nacional. Venezuela.

Quinteros Niria, Africano Nelly Y Faría Elisis (2008). "Clima organizacional y desempeño laboral del personal de la empresa vigilantes asociados costa oriental del lago. 
Rodríguez, D. (2004). Gestión Organizacional. Elementos para su estudio. Tercera Edición.

Salazar C. (2006) "Factores críticos que afectan el rendimiento laboral del personal académico y administrativo: aproximación a un enfoque metodológico para la medición del clima organizacional”. Universidad Simón Bolívar”. Universidad Central de Venezuela.

Sander, Benno (1990). Buenos Aires-Argentina. Educación, Administración y Calidad de Vida.

Stephen P. Robbins / Timothy A. Judge (2013). Comportamiento Organizacional. Editorial PEARSON - Decimoquinta Edición, México.

Téllez, Luís (2009). Universidad de Quindío. Sistema Integrado de Gestión. Generalidades de la Medición en el Sector Público- Los Indicadores. (Colombia) http://web2.uniquindío.edu.co/dep/plandes/documentos/sig/capacitaciones/indicadore s.pdf.

Uzcàtegui, Juan (2007) Dimensiones del Desempeño. http://macellum.blogspot.com/2007/09/dimensiones-del-desempeo.html.

Vásquez, S. (2007) "Nivel de motivación y su relación con la satisfacción laboral del profesional de enfermería en el Hospital Nacional Arzobispo Loayza" de la Universidad Nacional Mayor De San Marcos Facultad De Medicina Humana, Perú. 